\title{
A Diophantine inequality with four prime variables
}

\author{
Min Zhang* \& Jinjiang $\mathrm{Li}^{\dagger}$ \\ Department of Mathematics, China University of Mining and Technology ${ }^{* \dagger}$ \\ Beijing 100083, P. R. China
}

\begin{abstract}
Let $N$ be a sufficiently large real number. In this paper, it is proved that, for $1<c<\frac{1193}{889}$, the following Diophantine inequality

$$
\left|p_{1}^{c}+p_{2}^{c}+p_{3}^{c}+p_{4}^{c}-N\right|<\log ^{-1} N
$$

is solvable in prime variables $p_{1}, p_{2}, p_{3}, p_{4}$, which improves the result of $\mathrm{Mu}$ [14].

Keywords: Diophantine equation; Waring-Goldbach problem; prime variables; exponential sum
\end{abstract}

MR(2010) Subject Classification: 11J25, 11P32, 11P55, 11L07, 11L20

\section{Introduction and main result}

Let $k \geqslant 1$ be a fixed integer and $N$ a sufficiently large integer. The famous WaringGoldbach problem is to study the solvability of the following Diophantine equality

$$
N=p_{1}^{k}+p_{2}^{k}+\cdots+p_{r}^{k}
$$

in prime variables $p_{1}, p_{2}, \ldots, p_{k}$. For linear case, in 1937, Vinogradov [22] proved that every sufficiently large odd integer $N$ can be written as the sum of three primes. For $k=2$, in 1938, Hua [11] proved that the equation (1.1) is solvable for $r=5$ and sufficiently large integer $N$ satisfying $N \equiv 5(\bmod 24)$.

In 1952, Piatetski-Shapiro [15] studied the following analogue of the Waring-Goldbach problem: Suppose that $c>1$ is not an integer, $\varepsilon$ is a small positive number, and $N$ is a sufficiently large real number. Denote by $H(c)$ the smallest natural number $r$ such that the following Diophantine inequality

$$
\left|p_{1}^{c}+p_{2}^{c}+\cdots+p_{r}^{c}-N\right|<\varepsilon
$$

\footnotetext{
${ }^{\dagger}$ Corresponding author.

E-mail addresses: min.zhang.math@gmail.com (M. Zhang), jinjiang.li.math@gmail.com (J. Li).
} 
is solvable in primes $p_{1}, p_{2}, \ldots, p_{r}$, then it was proved in [15] that

$$
\limsup _{c \rightarrow+\infty} \frac{H(c)}{c \log c} \leqslant 4
$$

Also, in [15], Piatetski-Shapiro considered the case $r=5$ in (1.2) and proved that $H(c) \leqslant 5$ for $1<c<3 / 2$. Later, the upper bound $3 / 2$ for $H(c) \leqslant 5$ was improved successively to

$$
\frac{14142}{8923}, \quad \frac{1+\sqrt{5}}{2}, \quad \frac{81}{40}, \quad \frac{108}{53}, \quad 2.041, \quad \frac{665576}{319965}
$$

by Zhai and Cao [23], Garaev [8], Zhai and Cao [24], Shi and Liu [18], Baker and Weingartner [1], Zhang and $\mathrm{Li}$ [25], respectively.

From these results and Goldbach-Vinogradov theorem, it is reasonable to conjecture that if $c$ is near to 1 , then the Diophantine inequality (1.2) is solvable for $r=3$. This conjecture was first established by Tolev [20] for $1<c<\frac{27}{26}$. Since then, the range of $c$ was enlarged to

$$
\frac{15}{14}, \quad \frac{13}{12}, \quad \frac{11}{10}, \quad \frac{237}{214}, \quad \frac{61}{55}, \quad \frac{10}{9}, \quad \frac{43}{36}
$$

by Tolev [21], Cai [3], Cai [4] and Kumchev and Nedeva [12] independently, Cao and Zhai [6], Kumchev [13], Baker and Weingartner [2], Cai [5], successively and respectively.

Combining Tolev's method and the techniques of estimates on exponential sums of Fouvry and Iwaniec, in 2003, Zhai and Cao [23] proved that $H(c) \leqslant 4$ for $1<c<\frac{81}{68}$. Later, the range of $c$ for $H(c) \leqslant 4$ was enlarged to $1<c<\frac{97}{81}$ by $\mathrm{Mu}$ [14].

In this paper, motivated by [5], we shall continue to improve the result of $\mathrm{Mu}$ and establish the following theorem.

Theorem 1.1 Suppose that $1<c<\frac{1193}{889}$, then for any sufficiently large real number $N$, the following Diophantine inequality

$$
\left|p_{1}^{c}+p_{2}^{c}+p_{3}^{c}+p_{4}^{c}-N\right|<\log ^{-1} N
$$

is solvable in primes $p_{1}, p_{2}, p_{3}, p_{4}$.

Remark In order to compare our result with the results of $\mathrm{Mu}$ [14] and Zhai and Cao [23], we list the numerical result as follows

$$
\frac{1193}{889}=1.341957255 \cdots ; \quad \frac{97}{81}=1.197530864 \cdots ; \quad \frac{81}{68}=1.191176471 \cdots .
$$

Notation. Throughout this paper, we suppose that $1<c<\frac{1193}{889}$. Let $p$, with or without subscripts, always denote a prime number. $\eta$ always denotes an arbitrary 
small positive constant, which may not be the same at different occurrences; $N$ always denotes a sufficiently large real number. As usual, we use $\Lambda(n)$ to denote von Mangoldt's function; $e(x)=e^{2 \pi i x} ; f(x) \ll g(x)$ means that $f(x)=O(g(x)) ; f(x) \asymp g(x)$ means that $f(x) \ll g(x) \ll f(x)$.

We also define

$$
\begin{aligned}
& X=\frac{1}{2}\left(\frac{2 N}{5}\right)^{\frac{1}{c}}, \quad \varepsilon=\log ^{-2} X, \quad K=\log ^{10} X, \quad \tau=X^{1-c-\eta}, \\
& S(x)=\sum_{X<p \leqslant 2 X}(\log p) e\left(p^{c} x\right), \quad I(x)=\int_{X}^{2 X} e\left(t^{c} x\right) \mathrm{d} t, \quad \mathcal{T}(x)=\sum_{X<n \leqslant 2 X} e\left(n^{c} x\right) .
\end{aligned}
$$

\section{Preliminary Lemmas}

In this section, we shall give some preliminary lemmas, which are necessary in the proof of Theorem 1.1.

Lemma 2.1 Let $a, b$ be real numbers, $0<b<a / 4$, and let $r$ be a positive integer. Then there exists a function $\phi(y)$ which is $r$ times continuously differentiable and such that

$$
\left\{\begin{array}{cl}
\phi(y)=1, & \text { if } \quad|y| \leqslant a-b, \\
0<\phi(y)<1, & \text { if } \quad a-b<|y|<a+b \\
\phi(y)=0, & \text { if } \quad|y| \geqslant a+b,
\end{array}\right.
$$

and its Fourier transform

$$
\Phi(x)=\int_{-\infty}^{+\infty} e(-x y) \phi(y) \mathrm{d} y
$$

satisfies the inequality

$$
|\Phi(x)| \leqslant \min \left(2 a, \frac{1}{\pi|x|}, \frac{1}{\pi|x|}\left(\frac{r}{2 \pi|x| b}\right)^{r}\right) .
$$

Proof. See Piatetski-Shapiro [15] or Segal [17].

Lemma 2.2 Let $\mathcal{L}, \mathcal{Q} \geqslant 1$ and $z_{\ell}$ be complex numbers. Then we have

$$
\left|\sum_{\mathcal{L}<\ell \leqslant 2 \mathcal{L}} z_{\ell}\right|^{2} \leqslant\left(2+\frac{\mathcal{L}}{\mathcal{Q}}\right) \sum_{|q|<\mathcal{Q}}\left(1-\frac{|q|}{\mathcal{Q}}\right) \sum_{\mathcal{L}<\ell+q, \ell-q \leqslant 2 \mathcal{L}} z_{\ell+q} \overline{z_{\ell-q}} .
$$

Proof. See Lemma 2 of Fouvry and Iwaniec [7]. 
Lemma 2.3 Let $f(x)$ be a real differentiable function such that $f^{\prime}(x)$ is monotonic, and $\left|f^{\prime}(x)\right| \geqslant m>0$, throughout the interval $[a, b]$. Then we have

$$
\left|\int_{a}^{b} e^{i f(x)} \mathrm{d} x\right| \ll \frac{4}{m}
$$

Proof. See Lemma 4.2 of Titchmarsh [19].

Lemma 2.4 Suppose that $f(x):[a, b] \rightarrow \mathbb{R}$ has continuous derivatives of arbitrary order on $[a, b]$, where $1 \leqslant a<b \leqslant 2 a$. Suppose further that

$$
\left|f^{(j)}(x)\right| \asymp \lambda_{1} a^{1-j}, \quad j \geqslant 1, \quad x \in[a, b] .
$$

Then for any exponential pair $(\kappa, \lambda)$, we have

$$
\sum_{a<n \leqslant b} e(f(n)) \ll \lambda_{1}^{\kappa} a^{\lambda}+\lambda_{1}^{-1}
$$

Proof. See (3.3.4) of Graham and Kolesnik [9].

Lemma 2.5 For $1<c<2$, we have

$$
\begin{gathered}
\int_{\tau<|x|<K}\left|S^{2}(x) \Phi(x)\right| \mathrm{d} x \ll X^{1+\eta}, \\
\int_{\tau<|x|<K}\left|S^{4}(x) \Phi(x)\right| \mathrm{d} x \ll X^{4-c+\eta}, \\
\int_{-\tau}^{+\tau}|S(x)|^{2} \mathrm{~d} x \ll X^{2-c} \log ^{3} X, \\
\int_{-\tau}^{+\tau}|I(x)|^{2} \mathrm{~d} x \ll X^{2-c} \log ^{3} X .
\end{gathered}
$$

Proof. For (2.1) and (2.2), one can see Lemma 2.6 of Mu [14]. For (2.3) and (2.4), one can see Lemma 7 of Tolev [21].

Lemma 2.6 For $1<c<2$, then for $|x| \leqslant \tau$ we have

$$
S(x)=I(x)+O\left(X \exp \left(-(\log X)^{1 / 5}\right)\right) .
$$

Proof. See Lemma 4 of Zhai and Cao [23].

Lemma 2.7 For $1<c<2$, we have we have

$$
\int_{-\infty}^{+\infty} I^{4}(x) \Phi(x) e(-N x) \mathrm{d} x \gg \varepsilon X^{4-c} .
$$


Proof. See Lemma 8 of Zhai and Cao [23].

Lemma 2.8 Let $\alpha, \beta \in \mathbb{R}$ with $\alpha \beta(\alpha-1)(\beta-1)(\alpha-2)(\beta-2) \neq 0, F>0, M \geqslant 1, L \geqslant$ $1,\left|a_{m}\right| \leqslant 1,\left|b_{\ell}\right| \leqslant 1$. Then we have

$$
\begin{aligned}
& (F M L)^{-\eta} \cdot\left|\sum_{M<m \leqslant 2 M} \sum_{L<\ell \leqslant 2 L} a_{m} b_{\ell} e\left(F \frac{m^{\alpha} \ell^{\beta}}{M^{\alpha} L^{\beta}}\right)\right| \\
\ll & \left(F^{4} M^{31} L^{34}\right)^{\frac{1}{42}}+\left(F^{6} M^{53} L^{51}\right)^{\frac{1}{66}}+\left(F^{6} M^{46} L^{41}\right)^{\frac{1}{56}}+\left(F^{2} M^{38} L^{29}\right)^{\frac{1}{40}}+\left(F M^{9} L^{6}\right)^{\frac{1}{10}} \\
& +\left(F^{2} M^{7} L^{6}\right)^{\frac{1}{10}}+\left(F^{3} M^{43} L^{32}\right)^{\frac{1}{46}}+\left(F M^{6} L^{6}\right)^{\frac{1}{8}}+M^{\frac{1}{2}} L+M L^{\frac{1}{2}}+F^{-\frac{1}{2}} M L .
\end{aligned}
$$

Proof. See Theorem 9 of Sargos and Wu [16].

Lemma 2.9 Let $3<U<V<Z<X$ and suppose that $Z-\frac{1}{2} \in \mathbb{N}, X \gg Z^{2} U, Z \gg$ $U^{2}, V^{3} \gg X$. Assume further that $F(n)$ is a complex-valued function such that $|F(n)| \leqslant 1$. Then the sum

$$
\sum_{X<n \leqslant 2 X} \Lambda(n) F(n)
$$

can be written into $O\left(\log ^{10} X\right)$ sums, each of which either of Type I:

$$
\sum_{M<m \leqslant 2 M} a(m) \sum_{L<\ell \leqslant 2 L} F(m \ell)
$$

with $L \gg Z$, where $a(m) \ll m^{\eta}, M L \asymp X$, or of Type II:

$$
\sum_{M<m \leqslant 2 M} a(m) \sum_{L<\ell \leqslant 2 L} b(\ell) F(m \ell)
$$

with $U \ll M \ll V$, where $a(m) \ll m^{\eta}, b(\ell) \ll \ell^{\eta}, M L \asymp X$.

Proof. See Lemma 3 of Heath-Brown [10].

Lemma 2.10 Suppose that $\tau<|x|<K, M \ll X^{\frac{2971}{5334}}, a(m) \ll m^{\eta}, M L \asymp X$, then we have

$$
S_{I}(M, L):=\sum_{M<m \leqslant 2 M} \sum_{L<\ell \leqslant 2 L} a(m) e\left(x m^{c} \ell^{c}\right) \ll X^{\frac{2515}{2667}+\eta} .
$$

Proof. If $M \ll X^{\frac{4961}{10668}}$, then by Lemma 2.4 with the exponential pair $(\kappa, \lambda)=$ $A^{2} B(0,1)=\left(\frac{1}{14}, \frac{11}{14}\right)$, we deduce that

$$
\begin{aligned}
S_{I}(M, L) & \ll X^{\eta} \sum_{M<m \leqslant 2 M}\left|\sum_{L<\ell \leqslant 2 L} e\left(x m^{c} \ell^{c}\right)\right| \\
& \ll X^{\eta} \sum_{M<m \leqslant 2 M}\left(\left(|x| X^{c} L^{-1}\right)^{\frac{1}{14}} L^{\frac{11}{14}}+\frac{1}{|x| X^{c} L^{-1}}\right)
\end{aligned}
$$




$$
\begin{aligned}
& \ll X^{\eta}\left(K^{\frac{1}{14}} X^{\frac{c}{14}} M L^{\frac{5}{7}}+\tau^{-1} X^{1-c}\right) \\
& \ll X^{\frac{c}{14}+\frac{5}{7}+\eta} M^{\frac{2}{7}} \ll X^{\frac{2515}{2667}+\eta} .
\end{aligned}
$$

If $X^{\frac{4961}{10668}} \ll M \ll X^{\frac{2971}{5334}}$, then by Lemma 2.8 with $(m, \ell)=(m, \ell)$, we obtain

$$
S_{I}(M, L) \ll X^{\frac{2515}{2667}+\eta},
$$

which completes the proof of Lemma 2.10.

Lemma 2.11 Suppose that $\tau<|x|<K, X^{\frac{304}{2667}} \ll M \ll X^{\frac{1147}{2667}}, a(m) \ll m^{\eta}, b(\ell) \ll$ $\ell^{\eta}, M L \asymp X$. Then we have

$$
\mathcal{S}_{I I}(M, L):=\sum_{M<m \leqslant 2 M} \sum_{L<\ell \leqslant 2 L} a(m) b(\ell) e\left(x m^{c} \ell^{c}\right) \ll X^{\frac{2515}{2667}+\eta} .
$$

Proof. Taking $Q=X^{\frac{304}{2667}}(\log X)^{-1}$, if $X^{\frac{304}{2667}} \ll M \ll X^{\frac{1147}{2667}}$, by Cauchy's inequality and Lemma 2.2, we deduce that

$$
\begin{gathered}
S_{I I}(M, L) \ll\left(\sum_{L<\ell \leqslant 2 L}|b(\ell)|^{2}\right)^{\frac{1}{2}}\left(\sum_{L<\ell \leqslant 2 L}\left|\sum_{M<m \leqslant 2 M} a(m) e\left(x m^{c} \ell^{c}\right)\right|^{2}\right)^{\frac{1}{2}} \\
\ll L^{\frac{1}{2}+\eta}\left(\sum_{L<\ell \leqslant 2 L} \frac{M}{Q} \sum_{0 \leqslant q<Q}\left(1-\frac{q}{Q}\right)\right. \\
\left.\times \sum_{M+q<m \leqslant 2 M-q} a(m+q) \overline{a(m-q)} e\left(x \ell^{c}\left((m+q)^{c}-(m-q)^{c}\right)\right)\right)^{\frac{1}{2}} \\
\ll L^{\frac{1}{2}+\eta}\left(\frac { M } { Q } \sum _ { L < \ell \leqslant 2 L } \left(M^{1+\eta}+\sum_{1 \leqslant q<Q}\left(1-\frac{q}{Q}\right)\right.\right. \\
\left.\left.\times \sum_{M+q<m \leqslant 2 M-q} a(m+q) \overline{a(m-q)} e\left(x \ell^{c}\left((m+q)^{c}-(m-q)^{c}\right)\right)\right)\right)^{\frac{1}{2}} \\
\ll X^{\eta}\left(\frac{X^{2}}{Q}+\frac{X}{Q} \sum_{1 \leqslant q<Q} \sum_{M<m \leqslant 2 M}\left|\sum_{L<\ell \leqslant 2 L} e\left(x \ell^{c}\left((m+q)^{c}-(m-q)^{c}\right)\right)\right|\right)^{\frac{1}{2}} .
\end{gathered}
$$

Therefore, it is sufficient to estimate the inner sum

$$
\mathfrak{S}_{0}:=\sum_{L<\ell \leqslant 2 L} e\left(x \ell^{c}\left((m+q)^{c}-(m-q)^{c}\right)\right) .
$$

From Lemma 2.4 with the exponential pair $(\kappa, \lambda)=A B(0,1)=\left(\frac{1}{6}, \frac{2}{3}\right)$, we have

$$
\mathfrak{S}_{0} \ll\left(|x| X^{c-1} q\right)^{\frac{1}{6}} L^{\frac{2}{3}}+\frac{1}{|x| X^{c-1} q} .
$$


Putting the estimate (2.6) into (2.5), we deduce that

$$
\begin{aligned}
S_{I I}(M, L) & \ll X^{\eta}\left(\frac{X^{2}}{Q}+\frac{X}{Q} \sum_{1 \leqslant q<Q} \sum_{M<m \leqslant 2 M}\left(\left(|x| X^{c-1} q\right)^{\frac{1}{6}} L^{\frac{2}{3}}+\frac{1}{|x| X^{c-1} q}\right)\right)^{\frac{1}{2}} \\
& \ll X^{\eta}\left(\frac{X^{2}}{Q}+\frac{X}{Q}\left(K^{\frac{1}{6}} X^{\frac{c-1}{6}} L^{\frac{2}{3}} M Q^{\frac{7}{6}}+\tau^{-1} X^{1-c} M \log Q\right)\right)^{\frac{1}{2}} \\
& \ll\left(X^{2+\eta} Q^{-1}\right)^{\frac{1}{2}} \ll X^{\frac{2515}{2667}+\eta}
\end{aligned}
$$

which completes the proof of Lemma 2.11.

Lemma 2.12 For $1<c<\frac{1193}{889}$ and $\tau<|x|<K$, we have

$$
S(x) \ll X^{\frac{2515}{2667}+\eta} .
$$

Proof. Trivially, we have

$$
S(x)=\mathfrak{U}(x)+O\left(X^{1 / 2}\right),
$$

where

$$
\mathfrak{U}(x)=\sum_{X<n \leqslant 2 X} \Lambda(n) e\left(n^{c} x\right) .
$$

Taking $U=X^{\frac{304}{2667}}, V=X^{\frac{1147}{2667}}, Z=\left[X^{\frac{2363}{5334}}\right]+\frac{1}{2}$ in Lemma 2.9, it is not difficult to see that the sum

$$
\sum_{X<n \leqslant 2 X} \Lambda(n) e\left(n^{c} x\right)
$$

can be written into $O\left(\log ^{10} X\right)$ sums, each of which either of Type I:

$$
S_{I}(M, L)=\sum_{M<m \leqslant 2 M} \sum_{L<\ell \leqslant 2 L} a(m) e\left(x m^{c} \ell^{c}\right)
$$

with $L \gg Z, a(m) \ll m^{\eta}, M L \asymp X$, or of Type II:

$$
S_{I I}(M, L)=\sum_{M<m \leqslant 2 M} \sum_{L<\ell \leqslant 2 L} a(m) b(\ell) e\left(x m^{c} \ell^{c}\right)
$$

with $U \ll M \ll V, a(m) \ll m^{\eta}, b(\ell) \ll \ell^{\eta}, M L \asymp X$. For the sums of Type I, since $L \gg$ $Z$ and $M L \asymp X$, we get $M \ll X^{\frac{2971}{5334}}$. By Lemma 2.10, we have $S_{I}(M, L) \ll X^{\frac{2515}{2667}+\eta}$. For the sums of Type II, by Lemma 2.11, we get $S_{I I}(M, L) \ll X^{\frac{2515}{2667}+\eta}$. Thus, we deduce that

$$
\sum_{X<n \leqslant 2 X} \Lambda(n) e\left(n^{c} x\right) \ll X^{\frac{2515}{2667}+\eta} .
$$

From (2.7) and (2.8), we finish the proof of Lemma 2.12. 


\section{Proof of Theorem 1.1}

In this section, we use $\Phi(x)$ and $\phi(y)$ to denote the functions which appear in Lemma 2.1 with parameter $a=\frac{9 \varepsilon}{10}, b=\frac{\varepsilon}{10}, r=[\log X]$. Define

$$
\mathscr{B}_{4}(N)=\sum_{\substack{X<p_{1}, p_{2}, p_{3}, p_{4} \leqslant 2 X \\\left|p_{1}^{c}+\cdots+p_{4}^{c}-N\right|<\varepsilon}} \prod_{j=1}^{4} \log p_{j} .
$$

From the property of $\phi(y)$, we get $\mathscr{B}_{4}(N) \geqslant \mathscr{C}_{4}(N)$, where

$$
\mathscr{C}_{4}(N)=\sum_{X<p_{1}, p_{2}, p_{3}, p_{4} \leqslant 2 X}\left(\prod_{j=1}^{4} \log p_{j}\right) \phi\left(p_{1}^{c}+\cdots+p_{4}^{c}-N\right) .
$$

From the Fourier transformation formula, we derive that

$$
\begin{aligned}
\mathscr{C}_{4}(N) & =\sum_{X<p_{1}, \ldots, p_{4} \leqslant 2 X}\left(\prod_{j=1}^{4} \log p_{j}\right) \int_{-\infty}^{+\infty} e\left(\left(p_{1}^{c}+\cdots+p_{4}^{c}-N\right) y\right) \Phi(y) \mathrm{d} y \\
& =\int_{-\infty}^{+\infty} S^{4}(x) \Phi(x) e(-N x) \mathrm{d} x \\
& =\left(\int_{|x| \leqslant \tau}+\int_{\tau<|x|<K}+\int_{|x| \geqslant K}\right) S^{4}(x) \Phi(x) e(-N x) \mathrm{d} x \\
& =\mathscr{C}_{4}^{(1)}(N)+\mathscr{C}_{4}^{(2)}(N)+\mathscr{C}_{4}^{(3)}(N), \quad \text { say. }
\end{aligned}
$$

\subsection{The Estimate of $\mathscr{C}_{4}^{(1)}(N)$}

Define

$$
\begin{aligned}
& \mathscr{H}_{4}(N)=\int_{-\infty}^{+\infty} I^{4}(x) \Phi(x) e(-N x) \mathrm{d} x \\
& \mathscr{H}_{\tau}(N)=\int_{-\tau}^{+\tau} I^{4}(x) \Phi(x) e(-N x) \mathrm{d} x .
\end{aligned}
$$

From Lemma 2.1 and Lemma 2.3, we derive that

$$
\left|\mathscr{H}_{4}(N)-\mathscr{H}_{\tau}(N)\right| \ll \int_{\tau}^{\infty}|I(x)|^{4}|\Phi(x)| \mathrm{d} x \ll \varepsilon \int_{\tau}^{\infty}\left(\frac{1}{|x| X^{c-1}}\right)^{4} \mathrm{~d} x \ll \varepsilon X^{4-c-\eta} .
$$

From Lemma 2.5, Lemma 2.6 and the trivial estimate $S(x) \ll X$, we get

$$
\begin{aligned}
& \left|\mathscr{C}_{4}^{(1)}(N)-\mathscr{H}_{\tau}(N)\right| \leqslant \int_{-\tau}^{+\tau}\left|S^{4}(x)-I^{4}(x)\right||\Phi(x)| \mathrm{d} x \\
\ll & \varepsilon \cdot \int_{-\tau}^{+\tau}|S(x)-I(x)|\left(|S(x)|^{3}+|I(x)|^{3}\right) \mathrm{d} x
\end{aligned}
$$




$$
\begin{aligned}
& \ll \varepsilon \cdot X \exp \left(-(\log X)^{1 / 5}\right)\left(\int_{-\tau}^{+\tau}|S(x)|^{3} \mathrm{~d} x+\int_{-\tau}^{+\tau}|I(x)|^{3} \mathrm{~d} x\right) \\
& \ll \varepsilon X^{4-c} \exp \left(-(\log X)^{1 / 6}\right) .
\end{aligned}
$$

It follows from Lemma 2.7, (3.2) and (3.3) that

$$
\mathscr{C}_{4}^{(1)}(N)=\left(\mathscr{C}_{4}^{(1)}(N)-\mathscr{H}_{\tau}(N)\right)+\left(\mathscr{H}_{\tau}(N)-\mathscr{H}_{4}(N)\right)+\mathscr{H}_{4}(N) \gg \varepsilon X^{4-c} .
$$

\subsection{The Estimate of $\mathscr{C}_{4}^{(2)}(N)$}

According to the definition of $\mathscr{C}_{4}^{(2)}(N)$, we obtain

$$
\begin{aligned}
\left|\mathscr{C}_{4}^{(2)}(N)\right| & =\left|\sum_{X<p \leqslant 2 X}(\log p) \int_{\tau<|x|<K} e\left(p^{c} x\right) S^{3}(x) \Phi(x) e(-N x) \mathrm{d} x\right| \\
& \leqslant \sum_{X<p \leqslant 2 X}(\log p)\left|\int_{\tau<|x|<K} e\left(p^{c} x\right) S^{3}(x) \Phi(x) e(-N x) \mathrm{d} x\right| \\
& \ll(\log X) \sum_{X<n \leqslant 2 X}\left|\int_{\tau<|x|<K} e\left(n^{c} x\right) S^{3}(x) \Phi(x) e(-N x) \mathrm{d} x\right| .
\end{aligned}
$$

By Cauchy's inequality, we deduce that

$$
\begin{aligned}
& \left|\mathscr{C}_{4}^{(2)}(N)\right| \ll X^{\frac{1}{2}}(\log X)\left(\sum_{X<n \leqslant 2 X}\left|\int_{\tau<|x|<K} e\left(n^{c} x\right) S^{3}(x) \Phi(x) e(-N x) \mathrm{d} x\right|^{2}\right)^{\frac{1}{2}} \\
& =X^{\frac{1}{2}}(\log X)\left(\sum_{X<n \leqslant 2 X} \int_{\tau<|x|<K} e\left(n^{c} x\right) S^{3}(x) \Phi(x) e(-N x) \mathrm{d} x\right. \\
& \left.\times \int_{\tau<|y|<K} \overline{e\left(n^{c} y\right) S^{3}(y) \Phi(y) e(-N y)} \mathrm{d} y\right)^{\frac{1}{2}} \\
& =X^{\frac{1}{2}}(\log X)\left(\int_{\tau<|y|<K} \overline{S^{3}(y) \Phi(y) e(-N y)} \mathrm{d} y \int_{\tau<|x|<K} S^{3}(x) \Phi(x) e(-N x) \mathcal{T}(x-y) \mathrm{d} x\right)^{\frac{1}{2}} \\
& \ll X^{\frac{1}{2}}(\log X)\left(\int_{\tau<|y|<K}\left|S^{3}(y) \Phi(y)\right| \mathrm{d} y \int_{\tau<|x|<K}\left|S^{3}(x) \Phi(x) \mathcal{T}(x-y)\right| \mathrm{d} x\right)^{\frac{1}{2}} .
\end{aligned}
$$

For the inner integral in (3.5), we get

$$
\begin{aligned}
& \int_{\tau<|x|<K}\left|S^{3}(x) \Phi(x) \mathcal{T}(x-y)\right| \mathrm{d} x \\
\ll & \int_{\substack{\tau<|x|<K \\
|x-y| \leqslant X^{-c}}}\left|S^{3}(x) \Phi(x) \mathcal{T}(x-y)\right| \mathrm{d} x+\int_{\substack{\tau<|x|<K \\
X^{-c}<|x-y| \leqslant 2 K}}\left|S^{3}(x) \Phi(x) \mathcal{T}(x-y)\right| \mathrm{d} x .
\end{aligned}
$$


From Lemma 2.1, Lemma 2.12 and the trivial estimate $\mathcal{T}(x-y) \ll X$, we get

$$
\begin{gathered}
\int_{\substack{\tau<|x|<K \\
|x-y| \leqslant X^{-c}}}\left|S^{3}(x) \Phi(x) \mathcal{T}(x-y)\right| \mathrm{d} x \\
\ll \varepsilon X \times \sup _{\tau<|x|<K}|S(x)|^{3} \times \int_{\substack{\tau<|x|<K \\
|x-y| \leqslant X^{-c}}} \mathrm{~d} x \\
\ll \varepsilon X \cdot X^{\frac{2515}{889}-c+\eta} \ll \varepsilon X^{\frac{3404}{889}-c+\eta} .
\end{gathered}
$$

According to Lemma 2.4, for $X^{-c}<|x-y| \leqslant 2 K$, we get

$$
\begin{aligned}
\mathcal{T}(x-y) & \ll\left(|x-y| X^{c-1}\right)^{\kappa} X^{\lambda}+\frac{1}{|x-y| X^{c-1}} \\
& \ll|x-y|^{\kappa} X^{\kappa c+\lambda-\kappa}+\frac{1}{|x-y| X^{c-1}} .
\end{aligned}
$$

By choosing

$$
(\kappa, \lambda)=B A^{3} B A^{2} B A B A B A^{2} B A B A B(0,1)=\left(\frac{1731}{4492}, \frac{591}{1123}\right)
$$

in (3.8), we deduce that

$$
\mathcal{T}(x) \ll|x-y|^{\frac{1731}{4492}} X^{\frac{1731 c+633}{4492}}+\frac{1}{|x-y| X^{c-1}} .
$$

On the other hand, by Lemma 2.5 and Cauchy's inequality, we obtain

$$
\begin{aligned}
& \int_{\tau<|x|<K}\left|S^{3}(x) \Phi(x)\right| \mathrm{d} x \\
\ll & \left(\int_{\tau<|x|<K}\left|S^{2}(x) \Phi(x)\right| \mathrm{d} x\right)^{\frac{1}{2}}\left(\int_{\tau<|x|<K}\left|S^{4}(x) \Phi(x)\right| \mathrm{d} x\right)^{\frac{1}{2}} \\
\ll & \left(X^{1+\eta}\right)^{\frac{1}{2}} \cdot\left(X^{4-c+\eta}\right)^{\frac{1}{2}} \ll X^{\frac{5-c}{2}+\eta} .
\end{aligned}
$$

By (3.9), (3.10) and Lemma 2.12, we have

$$
\begin{aligned}
& \int_{\substack{\tau<|x|<K \\
X^{-c}<|x-y| \leqslant 2 K}}\left|S^{3}(x) \Phi(x) \mathcal{T}(x-y)\right| \mathrm{d} x \\
\ll & \int_{\substack{\tau<|x|<K \\
X^{-c}<|x-y| \leqslant 2 K}}\left|S^{3}(x) \Phi(x)\right|\left(|x-y|^{\frac{1731}{4492}} X^{\frac{1731 c+633}{4492}}+\frac{1}{|x-y| X^{c-1}}\right) \mathrm{d} x \\
\ll & X^{\frac{1731 c+633}{4492}+\eta} \int_{\tau<|x|<K}\left|S^{3}(x) \Phi(x)\right| \mathrm{d} x \\
& \quad+\varepsilon X^{1-c} \times \sup _{\tau<|x|<K}|S(x)|^{3} \times \int_{\begin{array}{c}
\tau<|x|<K \\
X^{-c}<|x-y| \leqslant 2 K
\end{array}} \frac{\mathrm{d} x}{|x-y|} \\
& \ll X^{\frac{1731 c+633}{4492}+\eta} \cdot X^{\frac{5-c}{2}+\eta}+\varepsilon X^{1-c} \cdot X^{\frac{2515}{889}+\eta} \\
\ll & X^{\frac{11863-515 c}{4492}+\eta}+\varepsilon X^{\frac{3404}{889}-c+\eta} \ll \varepsilon X^{\frac{3404}{889}-c+\eta} .
\end{aligned}
$$


From (3.6), (3.7) and (3.11), we get

$$
\int_{\tau<|x|<K}\left|S^{3}(x) \Phi(x) \mathcal{T}(x-y)\right| \mathrm{d} x \ll \varepsilon X^{\frac{3404}{889}-c+\eta},
$$

from which and (3.10), we can conclude that

$$
\left|\mathscr{C}_{4}^{(2)}(N)\right| \ll X^{\frac{1}{2}}(\log X)\left(X^{\frac{5-c}{2}+\eta} \cdot \varepsilon X^{\frac{3404}{889}-c+\eta}\right)^{\frac{1}{2}} \ll \varepsilon X^{4-c-\eta} .
$$

\subsection{The Estimate of $\mathscr{C}_{4}^{(3)}(N)$}

According to Lemma 2.1, we have

$$
\begin{aligned}
\left|\mathscr{C}_{4}^{(3)}(N)\right| & \ll \int_{K}^{\infty}|S(x)|^{4}|\Phi(x)| \mathrm{d} x \ll X^{4} \int_{K}^{\infty} \frac{1}{\pi|x|}\left(\frac{r}{2 \pi|x| b}\right)^{r} \mathrm{~d} x \\
& \ll X^{4}\left(\frac{r}{2 \pi b}\right)^{r} \int_{K}^{\infty} \frac{\mathrm{d} x}{x^{r+1}} \ll \frac{X^{4}}{r}\left(\frac{r}{2 \pi K b}\right)^{r} \\
& \ll \frac{X^{4}}{\log X} \cdot\left(\frac{1}{2 \pi \log ^{7} X}\right)^{\log X} \ll \frac{X^{4}}{X^{7 \log \log X+\log (2 \pi)} \ll 1 .}
\end{aligned}
$$

\subsection{Proof of Theorem 1.1}

From (3.1), (3.4), (3.12) and (3.13), we deduce that

$$
\mathscr{C}_{4}(N)=\mathscr{C}_{4}^{(1)}(N)+\mathscr{C}_{4}^{(2)}(N)+\mathscr{C}_{4}^{(3)}(N) \gg \varepsilon X^{4-c},
$$

and thus

$$
\mathscr{B}_{4}(N) \geqslant \mathscr{C}_{4}(N) \gg \varepsilon X^{4-c} \gg \frac{X^{4-c}}{\log ^{2} X}
$$

which completes the proof of Theorem 1.1.

\section{Acknowledgement}

The authors would like to express the most sincere gratitude to Professor Wenguang Zhai for his valuable advices and constant encouragement.

\section{References}

[1] R. Baker, A, Weingartner, Some applications of the double large sieve, Monatsh. Math. 170 (2013), no. 3-4, 261-304.

[2] R. Baker, A, Weingartner, A ternary Diophantine inequality over primes, Acta Arith. 162 (2014), no. 2, 159-196. 
[3] Y. C. Cai, A Diophantine inequality with prime variables, Acta Math. Sinica (Chin. Ser.), 39 (1996), no. 6, 733-742.

[4] Y. C. Cai, On a Diophantine inequality involving prime numbers. III, Acta Math. Sin. (Engl. Ser.), 15 (1999), no. 3, 387-394.

[5] Y. C. Cai, A ternary Diophantine inequality involving primes, Int. J. Number Theory, 14 (2018), no. 8, 2257-2268.

[6] X. D. Cao, W. G. Zhai, A Diophantine inequality with prime numbers, Acta Math. Sinica (Chin. Ser.), 45 (2002), no. 2, 361-370.

[7] E. Fouvry, H. Iwaniec, Exponential sums with monomials, J. Number Theory, 33 (1989), no. 3, 311-333.

[8] M. Z. Garaev, On the Waring-Goldbach problem with small non-integer exponent, Acta Arith., 108 (2003), no. 3, 297-302.

[9] S. W. Graham, G. Kolesnik, Van der Corput's Method of Exponential Sums, Cambridge University Press, New York, 1991.

[10] D. R. Heath-Brown, The Pjateckiı-S̆apiro prime number theorem, J. Number Theory, 16 (1983), no. 2, 242-266.

[11] L. K. Hua, Some results in the additive prime number theory, Quart. J. Math. Oxford Ser. (2), 9 (1938), no. 1, 68-80.

[12] A. Kumchev, T. Nedeva, On an equation with prime numbers, Acta Arith., 83 (1998), no. 2, 117-126.

[13] A. Kumchev, A Diophantine inequality involving prime powers, Acta Arith., 89 (1999), no. 4, 311-330.

[14] Q. W. Mu, On a Diophantine inequality over primes, Adv. Math. (China), 44 (2015), no. 4, 621-637.

[15] I. I. Piatetski-Shapiro, On a variant of Waring-Goldbachs problem, Mat. Sb., 30(72) (1952), no. 1, 105-120.

[16] P. Sargos, J. Wu, Multiple exponential sums with monomials and their applications in number theory, Acta Math. Hungar., 87 (2000), no. 4, 333-354.

[17] B. I. Segal, On a theorem analogous to Warings theorem, Dokl. Akad. Nauk SSSR (N. S.), 2 (1933), 47-49. 
[18] S. Y. Shi, L. Liu, On a Diophantine inequality involving prime powers, Monatsh. Math., 169 (2013), no. 3-4, 423-440.

[19] E. C. Titchmarsh, The Theory of the Riemann Zeta-Function, 2nd edn., Oxford University Press, Oxford, 1986.

[20] D. I. Tolev, Diophantine inequalities involving prime numbers, Ph.D. thesis, Moscow University, 1990.

[21] D. I. Tolev, On a Diophantine inequality involving prime numbers, Acta Arith., 61 (1992), no. 3, 289-306.

[22] I. M. Vinogradov, I. M. Vinogradov, Representation of an odd number as the sum of three primes, Dokl. Akad. Nauk. SSSR, 15 (1937), 291-294.

[23] W. G. Zhai, X. D. Cao, On a Diophantine inequality over primes, Adv. Math. (China), 32 (2003), no. 1, 63-73.

[24] W. G. Zhai, X. D. Cao, On a Diophantine Inequality Over Primes (II), Monatsh. Math., 150 (2007), no. 2, 173-179.

[25] M. Zhang, J. J. Li, On a Diophantine inequality with five prime variables, arXiv:1810.09368. 